\title{
Évaluer le développement durable : enjeux, méthodes, démarches d'acteurs
}

Compte-rendu de la journée d'étude du 3 novembre 2005, Lille

Bruno Villalba, Antoine Goxe et Jean-Christophe Lipovac

\author{
(2) OpenEdition \\ Journals \\ Édition électronique \\ URL : http://journals.openedition.org/developpementdurable/1676 \\ DOI : 10.4000/developpementdurable.1676 \\ ISSN : 1772-9971 \\ Éditeur \\ Association DD\&T \\ Référence électronique \\ Bruno Villalba, Antoine Goxe et Jean-Christophe Lipovac, «Évaluer le développement durable : enjeux, \\ méthodes, démarches d'acteurs », Développement durable et territoires [En ligne], Points de vue \\ (2003-2010), mis en ligne le 15 décembre 2005, consulté le 01 mai 2019. URL : http:// \\ journals.openedition.org/developpementdurable/1676; DOI : 10.4000/developpementdurable.1676
}

Ce document a été généré automatiquement le 1 mai 2019.

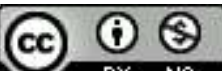

Développement Durable et Territoires est mis à disposition selon les termes de la licence Creative Commons Attribution - Pas d'Utilisation Commerciale 4.0 International. 


\title{
Évaluer le développement durable: enjeux, méthodes, démarches d'acteurs
}

\author{
Compte-rendu de la journée d'étude du 3 novembre 2005, Lille
}

\author{
Bruno Villalba, Antoine Goxe et Jean-Christophe Lipovac
}

1 Le 3 novembre 2005, le Centre Ressource du Développement Durable du Nord - Pas-deCalais (CERDD) ${ }^{1}$ et le réseau de recherche « Développement durable et territoires fragiles » organisaient une journée d'étude et de débat sur le thème : «Évaluer le développement durable : enjeux, méthodes, démarches d'acteurs ». Cette rencontre, qui bénéficiait du soutien du Conseil régional Nord - Pas-de-Calais, entendait contribuer à définir des outils d'aide à l'élaboration de politiques publiques locales de développement durable ${ }^{2}$. Cette manifestation avait pour objectif de faire un bilan et de tirer les enseignements de réflexions conceptuelles et d'expériences concrètes en matière d'évaluation du développement durable.

2 La journée s'est déroulée en deux temps: 1) La matinée a été réservée au partage de connaissances sur la problématique de l'évaluation en lien avec le développement durable ; 2) L'après-midi a été consacrée aux retours d'expériences : les méthodes et les outils, les dispositifs d'évaluation développés et expérimentés dans les collectivités locales.

Introduisant la journée, Alain Alpern (Vice-Président du Conseil régional Nord - Pas-deCalais, chargé du développement durable), présente d'une part la diversité et la richesse culturelle, naturelle et économique de la région Nord - Pas-de-Calais, mais souligne d'autre part que celle-ci constitue un exemple historique "parfait » de ce qu'est un développement non-durable ; sur ce territoire, la logique productiviste intensive (minière, industrielle, agricole) a eu pour résultante, par exemple, de voir ce territoire compter la moitié des sites et sols pollués recensés de France... Il rappelle que la Région s'est très tôt orientée vers cette problématique du développement durable, depuis l'action volontariste de M.-C. Blandin à la présidence de la région de 1992 à 1998. Il souligne également l'investissement institutionnel, notamment en ce qui concerne le soutien aux agendas 21 locaux des collectivités infra-régionale avec le programme « PRASA 21 » (une quarantaine 
de collectivités engagées, ce qui place l'espace régional en première position au niveau national), le soutien à la création et au fonctionnement de structures associatives d'animation et d'expertise (CERDD, CD2E $\mathrm{E}^{3}$ ), mais aussi l'essaimage progressif de cette thématique depuis quelques années (par exemple avec l'élaboration d'agendas 21 de lycées, etc.).

4 Face à cet effort, l'évaluation constitue une priorité : si l'on suit certains textes de références (dont le programme de recommandations Action 21 adopté à Rio), elle est devenue une thématique consubstantielle de la problématique du développement durable. Des réflexions sur les indicateurs ont été menées, mais il reste encore beaucoup de travail et d'expérimentations à mener, et sur la conceptualisation, et sur l'application concrète de ces indicateurs (par exemple l'« épargne authentique », l'« empreinte écologique », etc.). Il faut par ailleurs faire un travail important de mobilisation des acteurs; en effet, le principe de l'évaluation semble devoir se coupler avec le principe de la gouvernance (participation des différents acteurs concernés ou impliqués au processus de choix); l'évaluation se fait ainsi participative, tenant compte des avancées méthodologiques (conférences de consensus, conférences de citoyens) et/ou construisant des démarches d'acteurs originales (comme dans le cadre de l'élaboration du contrat d'agglomération de la Communauté Urbaine de Dunkerque par exemple).

Les enjeux, méthodes et démarches d'acteurs constituent ainsi le cœur de cette journée.

Bertrand Zuindeau (co-animateur du réseau Développement durable et territoires fragiles) intervient ensuite pour présenter l'optique plus heuristique des réflexions du réseau pluridisciplinaire de recherche «DDTF » sur la question de l'évaluation (notamment autour du travail d'analyse et de capitalisation ${ }^{4}$ ). Cette question de l'évaluation et de ses différentes approches théoriques et pratiques constitue en effet un des axes de la programmation scientifique du réseau DDTF (qu'il s'agisse des méthodes d'évaluation monétaire, ou d'évaluation multi-critères en économie, ou dans une approche sociopolitique sur l'élaboration des instruments d'évaluation de l'action publique et leurs usages par les acteurs).

7 Après cette double introduction, la matinée était consacrée aux présentations de trois intervenants :

- Magali Bardou, du Ministère de l'Ecologie et du Développement Durable sur l'élaboration d'un cadre de référence pour la reconnaissance des démarches locales de développement durable, chantier mené sous l'égide de la Délégation Interministérielle sur le Développement Durable,

- Aurélien Boutaud, docteur en sciences et génie de l'environnement de l'Ecole nationale des Mines de Saint-Etienne et consultant, présentant les grandes lignes d'analyse développée dans sa thèse sur les « Outils d'Analyse et de Questionnement au regard du Développement Durable » (OQADD) développés par les acteurs locaux,

- Jacques Theys, Directeur du Centre de Prospective et de Veille Scientifique (CPVS) du Ministère de l'Equipement, pour une mise en perspective critique des acquis des réflexions scientifiques et des expérimentations pratiques en matière d'évaluation du développement durable. 


\section{Evaluer un projet de territoire au regard du développement durable : un cadre de référence à partager}

Magali Bardou présente l'état des réflexions et des analyses menées et animées par le Bureau de la ville du MEDD ${ }^{5}$ sur la question de l'évaluation d'un " projet de territoire, des enjeux de développement durable à la démarche de projet. » Elle rappelle dans un premier temps l'évolution institutionnelle ministérielle vis-à-vis de cette question. Le Ministère de l'environnement travaille depuis une dizaine d'années sur la question de l'animation des territoires (depuis les Plans municipaux d'environnement - PME, créés en 1988 pendant le ministère Lalonde, puis les Chartes d'environnement lancées en 1994 en réponse aux préconisations de l'Agenda 21 de Rio), mais en étant davantage centré sur la question environnementale. Les appels à projets « outils et démarches en vue de la réalisation des A21 locaux ", lancés à trois reprises en1997, 2000 et 2003, s'inscrivent dans cette lignée, en tentant de dégager des expériences exemplaires à capitaliser et des principes d'action plus transversaux pour les projets de territoire. L'objectif est de rassembler des informations concrètes pour se forger un référentiel. La Stratégie nationale du développement durable (SNDD), adoptée en juin 2003, développe une approche plus volontariste et incitative (projets territoriaux de développement durable), en fixant notamment un objectif de soutien à 500 Agendas 21 locaux effectifs à l'horizon 2008. Mais au-delà de l'affichage et de l'objectif quantitatif, l'enjeu est de forger un cadre de référence national permettant de donner un contenu lisible, reconnu et adopté par le plus grand nombre, qui offrirait aux collectivités territoriales des orientations et des pistes d'actions plus précises, des exemples concrets d'expériences, et permettrait d'apprécier ou d'évaluer des projets en cours ou en phase de démarrage. A terme, il s'agit de mettre en place un processus de reconnaissance au niveau national pour valoriser les "bons " projets à l'heure où la thématique du développement durable est devenue un argument de promotion du territoire et court le risque de se réduire à un slogan. Il va donc falloir évaluer la qualité des projets présentés : s'agit-il vraiment des projets de développement durable? La question des bases communes se pose donc.

Ce cadre de référence a été élaboré en concertation avec le plus grand nombre de personnes possibles, en adoptant une démarche transversale aux différents services de l'Etat (travail en comité technique, via les Hauts fonctionnaires au DD dans chaque ministère), une élaboration partenariale et ouverte, via un comité ressources ${ }^{6}$ et un comité de pilotage national "Agendas 21 locaux" largement ouvert aux acteurs institutionnels, économiques et associatifs. Ce travail hérite des réflexions sur les Chartes d'environnement et sur les appels à projet « outils et méthodes en vue de la réalisation des A21 locaux ».

10 La logique du cadre de référence qui finit par émerger tourne autour de l'idée qu'il convient de concilier l'appréciation du contenu (une stratégie d'action répondant aux cinq finalités du développement durable ... et s'exprimant dans divers domaines: santé, habitat, urbanisme, gestion des ressources, accès à la connaissance...) et de tenir compte de la méthode utilisée (5 éléments déterminants: participation, transversalité, stratégie d'amélioration, organisation de la décision, évaluation continue). 
11 Les projets territoriaux doivent répondre à cinq finalités dégagées par le travail de la Délégation interministérielle au développement durable: lutter contre le changement climatique et protéger l'atmosphère ; préserver la biodiversité, protéger les milieux et les ressources ; permettre l'épanouissement de tous les êtres humains ; assurer la cohésion sociale, et la solidarité entre territoires et entre générations; fonder les dynamiques de développement sur des modes de production et de consommation responsables. Ces finalités sont transversales aux trois (ou quatre en intégrant la culture ${ }^{7}$ ) «piliers » du développement durable, tout en indiquant des ambitions politiques plus claires. Toute action ou projet de développement durable se doit d'y contribuer positivement, et surtout de ne menacer aucune d'entre elles. Ces finalités doivent pouvoir être déclinées au niveau des territoires, dans tous les domaines d'intervention (Solidarité et coopération décentralisée, urbanisme, habitat, logement, accès à la connaissance, etc.) La démarche doit aussi s'appuyer sur cinq éléments déterminants (une stratégie d'amélioration continue, qui repose sur: la participation des acteurs; l'évaluation en continu; la transversalité ; l'organisation du pilotage technique et politique).

En ce qui concerne l'évaluation, la délégation interministérielle au développement durable insiste sur la difficulté de l'élaborer, de la maintenir dans le temps et de la rendre utile à l'opération en cours. Trois orientations principales sont cependant retenues :

- définir une méthode avec les acteurs (associer les acteurs dès les premières étapes, définir une méthode en amont, se donner les moyens de l'évaluation);

- Préciser les objectifs de l'évaluation (mesurer les améliorations, vérifier la cohérence et la pertinence)

- et enfin, rendre compte et permettre l'évolution du projet en gardant sa cohérence (communiquer et nourrir l'avenir du projet).

13 Ce document doit être discuté par les acteurs, afin de faciliter son appropriation. Il prend aussi appui sur un dispositif expérimental en 2005. Ce dispositif a pour objectif de tester, avec les territoires, le cadre de référence (manques, facilité de compréhension, reformulations, utilité...). Il doit permettre l'élaboration d'une grille d'évaluation des projets fondée sur ce cadre et préparer les modalités d'une reconnaissance nationale des projets territoriaux de développement durable. Pour les collectivités, il serait intéressant que cette référence permette d'évaluer les démarches déjà entreprises au regard du développement durable et d'appréhender les marges de progrès pour mettre en place un projet plus global de type Agenda 21 ; de mettre en évidence, dans le cas d'une démarche globale déjà avancée (projet/contrat d'agglomération ou de pays...), la façon dont cette démarche satisfait aux éléments de cadrage (finalités - éléments de démarche), et ainsi d'élaborer une grille de questionnement adaptée aux différents maîtres d'ouvrage permettant de vérifier la cohérence des réponses apportées aux questions et projets « ordinaires ».

14 Les perspectives s'orientent vers une «dd-conditionnalité» des aides publiques et des contractualisations, point qui a été abordé lors du second séminaire gouvernemental sur le développement durable de mars 2005, ou bien encore vers des procédures d'évaluationaudit des projets à l'étape de leur mise en œuvre (de l'évaluation ex ante, comme elle a été conduite dans le cadre de la sélection des lauréats des appels à projets «outils et démarches » de 1997, 2000 et 2003, à l'évaluation de la mise en œuvre). Mais, comme le précise Magali Bardou, "pour l'instant, nous ne sommes sûrs de rien. Mais à partir du moment où l'on contractualise avec ce cadre de référence, on conditionne les aides au développement durable. Plus on arrivera à faire s'entendre un maximum d'acteurs sur l'importance de ce cadre de 
référence, plus on sera en mesure d'inciter à ce que les aides aux territoires soient orientées autour de ces questions. " Concernant les conditions qui permettent d'apprécier le contenu des projets (notamment autour des cinq critères), l'approche estime que les critères ne sont pas de même niveau, mais sans qu'ils soient à hiérarchiser a priori (un projet territorial est d'abord une réflexion sur les enjeux de son territoire); se pose alors la question de savoir comment l'on définit une priorité et comment on l'insère dans les enjeux globaux ? Cependant, Magali Bardou rappelle que les projets territoriaux doivent s'inscrire dans une dynamique de solidarité inter-territoriale (par rapport aux enjeux globaux, par rapport aux autres territoires en tenant compte des découpages administratifs, des projets nationaux, etc.).

\section{Evaluation du développement durable : du global au local. De quels outils les collectivités locales disposent-elles?}

Après un rapide rappel historique sur les origines de la notion de développement durable, Aurélien Boutaud ${ }^{8}$ porte son attention sur les dispositifs mis en place par les acteurs publics afin d'intégrer le développement durable dans leurs politiques. Il constate tout d'abord que ces démarches normatives (indicateurs, grilles d'analyse des politiques) ont l'avantage de nous fournir des informations très précises sur la vision que les acteurs publics se font du développement durable. Il analyse ensuite le positionnement de ces outils dans les processus de décision, qui permet de saisir l'importance accordée au développement durable par les acteurs publics. Les outils d'évaluation du développement durable fournissent des informations particulièrement précieuses sur la manière dont les politiques publiques traduisent effectivement en actes cette problématique.

Parmi ces outils d'évaluation, les "Outils de Questionnement et d'Analyse des projets (ou politiques) en matière de Développement Durable » (OQADD $)^{9}$, souvent appelés par les praticiens " grilles développement durable ", sont des objets créés soit pour évaluer des projets dans le cadre d'appels d'offre, soit pour aider les porteurs de projet d'un territoire à mieux prendre en compte les critères du développement durable, soit encore pour rendre le concept plus facilement assimilable par les personnels administratifs. Développés sur le terrain par des acteurs publics, des associations ou éventuellement des bureaux d'études, ces OQADD sont des dispositifs dont la finalité première consiste à évaluer, à analyser ou plus simplement à questionner des politiques ou des projets (dont l'état d'avancement est variable) par rapport aux exigences du développement durable, et dont la forme est le plus souvent celle d'une grille de critères, organisés et hiérarchisés en arborescence, et formulés sous forme de questions dont les résultats peuvent être (mais ne sont pas nécessairement) agrégés et pondérés entre eux. Les OQADD sont donc des "grilles de critères » qui ont vocation à aider les porteurs de projets (ou de politiques) à prendre en compte le développement durable dans leur démarche.

17 Aurélien Boutaud a ainsi identifié 684 critères différents dans l'ensemble de ces OQADD, qui se sont avérés être répartis de manière très équilibrée entre les trois « sphères » du développement durable $(1:$ critères sociaux, $2:$ critères environnementaux et $3:$ critères économiques). Cela témoigne d'une « approche consensuelle » du DD, mais qui se heurte à une appréciation plus complexe de la réalité (déséquilibres entre territoires, asymétries des orientations économiques, etc.) Il estime que «la vision du développement durable 
adoptée par les acteurs publics ne permet pas forcément de véritablement "penser le changement". »

Il poursuit son analyse en s'interrogeant sur le degré d'intégration du développement durable (même dans sa version « allégée ») dans les politiques publiques. L'enquête qu'il a menée en 2004 auprès d'un certain nombre de praticiens ${ }^{10}$ apporte quelques éléments de réponse.

Si les OQADD sont des outils propices à transformer certaines pratiques (créer une vision et une culture commune, rassurer et « décomplexer » les acteurs face à cette problématique du développement durable, sensibiliser, informer, communiquer, etc.), ils présentent aussi certains inconvénients (subjectivité des évaluateurs, difficulté de trouver un équilibre entre universalité des thèmes à aborder et particularité des cas à traiter, passer du constat critique à l'action constructive, etc.) Au final, il estime que «les OQADD jouent souvent le rôle de 'poil à gratter', obligeant les responsables techniques et politiques à réinterroger leurs modes de fonctionnement. Et cette remise en cause n'est pas toujours appréciée, ce qui explique sans doute pourquoi un certain nombre de démarches ont été soit abandonnées, soit laissées en jachère... soit très édulcorées. (...) L'analyse du fonctionnement des OQADD nous montre en effet à quel point le développement durable n'est pas encore considéré comme une valeur politique dominante.» Par conséquent, reprenant une expression d'Olivier Godard ${ }^{11}$ il plaide pour une approche "non-fractale » (non-équilibrée) du développement durable, permettant de gérer les déséquilibres et instaurer des rapports plus solidaires, notamment à l'échelle mondiale. En effet, si la «plus-value » du développement durable au niveau théorique semble résider dans l'attention portée au « global » et au long terme, soit aux acteurs « faibles » non présents car éloignés ou à venir (les fameuses générations futures), ces perspectives spatiales et temporelles élargies semblent trop largement oubliées dans les processus du prise de décision. Finalement, les OQADD ne peuvent avoir qu'un impact que limité dans la mesure où ils n'infléchissent que rarement les logiques mêmes des orientations générales du développement des territoires, des décisions les plus amont. Ils apparaissent ainsi comme des " pots catalytiques administratifs » plus ou moins efficaces élaborés et/ou utilisés par les services pour tenter de rendre «plus durables » des décisions trop souvent non interrogées elles-mêmes.

\section{Evaluer le développement durable : où en est-on ?}

20 A la suite de ces deux premières interventions, Jacques Theys ${ }^{12}$ exprime ses doutes sur l'importance de constituer des référentiels complexes sur le développement durable. Il estime que, si l'évaluation prend de plus en plus d'importance dans les pratiques administratives en France, et notamment sur la question de la durabilité, et que la demande d'évaluation est quantitativement de plus en plus forte, les outils restent en revanche un peu faibles.

Pour l'instant, de nombreux obstacles pèsent sur le développement de l'évaluation :

- obstacle politique (difficulté de faire du développement durable un véritable enjeu territorial local - effet d'emboîtement des territoires) ;

- obstacle culturel (la France souffre d'un retard culturel face aux politiques d'évaluation, de face à face ; le suffrage universel constitue la forme d'évaluation de l'action publique quasiunique dans la culture politico-administrative française. En témoigne l'abandon par le 
Commissariat Général du Plan de sa mission d'évaluation des politiques publiques, notamment des Contrats de Plan Etat-Région);

- institutionnel (les incitations pour développer l'évaluation ne sont pas importantes -elles sont plus fortes du côté des entreprises, avec les agences de notation par exemple);

- obstacle de la disponibilité des statistiques (un coût d'accès à l'information, le manque d'autonomie des collectivités territoriales pour la construction des données, etc.), et méthodologique (incapacité à stabiliser un véritable référentiel partagé sur le développement durable ; est-ce que l'on peut s'accorder sur des priorités ?).

Par conséquent, sauf exception (comme la Région Nord-Pas de Calais ou la communauté urbaine de Dunkerque), nous ne construisons que des évaluations ponctuelles, qui recyclent d'autres informations, qui sont assez peu intégrées à la décision, mais aussi peu débattues et diffusées dans le public et finalement, peu partagées entre les différentes collectivités territoriales. Il y a une grande difficulté, en particulier, à stabiliser les observatoires, les systèmes d'observation ; l'énergie dépensée à construire le système, les indicateurs qui le constituent, etc. n'est généralement pas réinvestie dans l'utilisation du système. Pour ponctuer ce constat amère d'une formule lapidaire, Jacques Theys conclut que « les indicateurs sont trop souvent des cimetières » précise-t-il.

Par rapport à cette situation, Jacques Theys estime qu'il faut abandonner l'idée que, entre l'écart de la demande et de l'offre, il existe un système unique, qui puisse répondre à toutes les demandes et qui soit durable dans le temps. L'important consiste à savoir ce que l'on souhaite, quelle est la finalité du projet, de la politique, et comment s'outiller en conséquence. Quatre grandes utilisations de l'évaluation peuvent être construites selon lui :

- l'évaluation comme un outil de suivi d'une stratégie (a);

- l'évaluation (indicateurs, etc.) comme outil de dialogue et mobilisation des acteurs (b) ;

- l'évaluation comme outil de positionnement (par rapport aux autres: cela concerne la question de référentiel, de positionnement des territoires et leurs contradictions) (c) ;

- l'évaluation - et c'est le domaine le plus difficile - comme outil de diagnostic (savoir si on est ou pas dans des politiques contribuant de manière concrète et effective à un développement durable) (d).

Dans ces quatre perspectives, comment faire fonctionner une politique d'évaluation?

a) Mettre en place des indicateurs pour tester ce suivi, cela n'est pas principalement une question de méthode; cela suppose quatre conditions admises: 1) l'expression claire d'une politique ; 2) une politique avec peu d'objectifs (moins de 10, et à hiérarchiser) ; 3 ) des conditions corrélées avec les projets retenus (les critères retenus se retrouvent dans les politiques sélectionnées - articuler les deux niveaux, entre les projets et les politiques générales) ; 4) un travail d'interprétation des résultats (une évaluation des politiques publiques au sens classique).

b) La deuxième perspective, totalement différente de la première, «et sans doute la plus intéressante", consiste à considérer l'évaluation comme un outil de mobilisation, de dialogue entre les électeurs (les publics), les collectivités locales, et les acteurs faibles (ceux en dehors de la collectivité, absents) ; cela suppose qu'il y ait une co-construction des indicateurs avec la population concernée et de rendre compte périodiquement du suivi et de l'évolution de ces indicateurs (évaluation annuelle, par exemple via les budgets municipaux - comme aux Pays-Bas -, ou ponctuelles). Au niveau du contenu, ces indicateurs ( 20 ou 30 au maximum pour qu'ils puissent rester lisibles et compréhensibles 
ensemble) qui permettent le dialogue doivent être basés sur quatre principes pour être efficaces dans cette perspective de mobilisation :

- qu'ils permettent de voir l'impact des activités locales sur le monde extérieur (comme l'empreinte écologique) ;

- voir ce que fait la collectivité (investissements locaux) ;

- voir en quoi la population est concernée par certains problèmes (comme les facteurs d'inégalités écologiques);

- que les indicateurs qui permettent à chacun de voir comment il peut agir (transport, recyclage, etc.) à son niveau, au quotidien.

On peut ainsi concevoir une logique de dialogue, continue ou régulière, avec la population et de mobilisation.

c) La troisième optique change encore de perspective ; elle correspond à la manière dont un territoire va se positionner face aux autres territoires. Cela recouvre trois aspects :

- communiquer sur ce que l'on fait ;

- se comparer ;

- se conformer (par rapport aux exigences nationales et internationales).

Ce que l'on voit c'est la contradiction entre ces différents outils, et donc la nécessité de réguler, selon certaines modalités différentes de régulation :

- régulation compétitive (dans la logique des agences de notation par exemple);

- régulation normative (un cadre est défini au niveau national et international, dans une logique top down ou bottom up; mais ces expériences sont pour l'instant peu concluantes du fait des difficultés à imposer ces indicateurs, comme constaté pour les tentatives de construction d'indicateurs de la DATAR, ou de l'Europe);

- enfin la régulation coopérative, en s'inspirant par exemple de l'ARPE ${ }^{13}$ et du réseau des villes durables qui s'est constitué en Midi-Pyrénées ;

Un cadre de référence normatif est souvent global et peu adapté aux situations locales, ce qui suppose de valoriser la démarche coopérative - une grille d'évaluation minimale, mais avec un système de label.

d) Enfin, la perspective liée à l'évaluation comme outil de diagnostic. Cela pose la question de savoir à quel objectif central répond le développement durable. Pour l'instant, il n'y a pas de consensus sur ce que l'on veut mesurer (l'environnement?). Il y a trois choses possibles à réaliser : 1) un effort de réinvestissement scientifique (on doit l'interpréter d'une manière plus extensive, car ce concept reste trop largement "sous-exploité ", autour de l'articulation des trois dimensions environnementales, économiques et sociales): comment se mettre d'accord sur une grammaire commune? Comment concilier les différentes visions du développement durable? Comment analyser aussi la liaison avec les questions du développement ou bien encore les questions des indicateurs (comme l'empreinte écologique) ?2) A court terme, l'évaluation peut se construire autour de méthodes de hiérarchisation autour d'un territoire notamment en s'intéressant en priorité au niveau du territoire sur les aspects «non durables» du mode de développement. Cela doit nécessairement se faire autour de questions de prospective (s'interroger sur le long terme, les dynamiques territoriales de long terme), qui semblent bien impératives lorsqu'on veut placer son action dans une perspective de développement durable. 3) Et à moyen terme - et c'est le rôle des observatoires - il faut faire un travail de capitalisation de certains indicateurs (empreinte écologique, inégalité écologique, etc.). 

les « 3 piliers", qui constitue à ses yeux une impasse scientifique et en termes de construction des connaissances. Le travail de l'IFEN sur les indicateurs de développement durable, engagé depuis plusieurs années, a ainsi été écarté au motif que le domaine de compétence de l'Institut se limite à l'environnement. La dimension intégratrice et transversale du développement durable, qui s'efface dans la représentation sectorisée en " piliers ", se heurte ainsi également à ce niveau à la sectorisation et aux concurrences entre administrations : aujourd'hui, au niveau de la statistique nationale, le travail sur les indicateurs de développement durable est abandonné; les indicateurs touchant aux questions sociales sont construits et instruits par la DARES ${ }^{14}$ et l'INSEE, les indicateurs économiques par l'INSEE, et les indicateurs environnementaux par l'IFEN... Le recul est donc très net sur cette question, mais qui croit sérieusement que la juxtaposition de ces travaux est pertinente?

\section{Quels sont les enjeux stratégiques de l'évaluation au regard du développement durable?}

34 A la suite de ces trois interventions, une table-ronde, animée comme l'ensemble de la matinée par Bertrand Zuindeau, s'est déroulée autour de la question suivante : "Quels sont les enjeux stratégiques de l'évaluation au regard du développement durable? ", avec Valérie Caron, Responsable de cabinet, Mairie de Loos en Gohelle; André Colin, Gérant de la coopérative de conseil en développement durable Extra-Muros ; Pascal Dubois, Directeur de cabinet du Maire de Valenciennes; Sylvie Depraetere, Responsable de la Mission Animation et Ingénierie du Développement Durable, Direction de la Prospective, du Plan et de l'Evaluation au Conseil Régional Nord - Pas-de-Calais; Sandrine Rousseau, Economiste à l'Université de Lille 1, et Bruno Villalba, Politiste à l'Université de Lille 2.

Cette table-ronde a permis des réactions et une mise en perspective par différents acteurs de la région des interventions de la matinée, et la présentation d'expériences menées au niveau de quelques collectivités locales. Sans vouloir prétendre à l'exhaustivité, signalons quelques réflexions proposées par ces acteurs :

- Valérie Caron a insisté sur le caractère « pragmatique » et " pro-actif » de la mairie de Loos-en-Gohelle. Si les questionnements théoriques sont souvent loin des acteurs des collectivités, particulièrement des communes petites et moyennes, les expérimentations et innovations sont le reflet d'un volontarisme politique relayé par les services. Un des soucis est que les collectivités ont encore peu d'expériences sur lesquelles s'appuyer, mais il s'agit d'avancer malgré tout, nous sommes constamment dans un processus d'innovation qui appelle une évaluation en continue. A Loos-en-Gohelle, on formalise de manière mnémotechnique la démarche par le sigle «EEG»: «expérimentation, évaluation, généralisation ». Notre démarche s'inscrit d'emblée dans une approche participative. Il s'agit de répondre aux besoins de la population. 
37 Parmi les expériences d'évaluation déjà abordées, nous travaillons sur l'évaluation des réalisations en Haute Qualité Environnementale sur la commune, sur l'évaluation des dispositifs de concertation et de participation dans l'optique d'avancer sur la question des indicateurs de développement humain, enfin, sur le calcul de l'empreinte écologique de la ville à la fois comme outil de comparaison et outil pédagogique auprès des acteurs locaux. Pour finir, je soulignerais que nous rentrons dans un processus d'évaluation dans la perspective également de mutualiser des pratiques et de favoriser le transfert de savoirfaire.

39 - Pascal Dubois, de la mairie de Valenciennes, a pour sa part voulu revenir sur la question du consensus, qui constitue un impératif pour agir de manière efficace au niveau d'un territoire avec les différents partenaires et les habitants. Il importe de partager un langage, de se comprendre entre acteurs. La construction du consensus n'implique pas l'absence de débats, mais doit permettre d'une part un travail de communication et de pédagogie autour des projets, et la prise en compte au sérieux des demandes et critiques exprimées, ce pour rester dans une dynamique constructive. A Valenciennes, nous réfléchissons depuis 10 ans sur un nouveau type de développement à partir d'un développement non durable et de nombreuses séquelles importantes ( 150 hectares fiches industrielles, taux de chômage très élevé ... ). Après un diagnostic de l'existant et des constats de non durabilité, au bout de 10 ans de réflexions, nous aboutissons à une ville en chantier. Nous avons fait le choix de mener un projet en concertation avec la population. Notre objectif est de construire un projet concertée mais aussi d'en assurer le suivi. Aujourd'hui, concrètement sur un projet d'aménagement de l'espace public, il s'agit de mettre en place une information permanente sur la réalisation des travaux par exemple. La participation joue un rôle de première importance : répondre aux besoins des habitants et faire comprendre les enjeux et les objectifs du développement durable.

L'évaluation est nécessaire pour s'assurer que l'on répond aux exigences du développement durable (en particulier des exigences du développement urbain durable telles que définies lors de la Conférence de Lisbonne) et que l'on s'inscrive dans un processus d'amélioration continue ( nous avons écrit un second plan d'action agenda 21 en travaillant avec la population à partir des 27 indicateurs de l'Agence Régional de l'Environnement Rhône-Alpes).

41 - Enfin, concluant un premier tour de table des acteurs des collectivités territoriales, Sylvie Depraetere est revenue sur les enseignements tirés des expériences d'évaluation au sein du Conseil Régional. Tout d'abord, le changement apparaît plus particulièrement dans la manière de construire les méthodes et les indicateurs, car la démarche suivie, notamment pour la construction d'indicateurs de développement durable dans le cadre du Contrat de Plan Etat-Région, a été réalisée en inter-services ce qui a permis une amorce de transversalité, de réflexion et d'appropriation collectives autour du développement durable. Cela implique une forme de compromis entre la qualité "scientifique " et la manière dont on gère le dialogue et la co-construction. Le second point, souligné par Sylvie Depraetere, est celui de la volonté. Le choix des techniques doit également intégrer la lisibilité et la pertinence au regard des choix opérés par les décideurs, dans le cas présent des élus. Les indicateurs doivent être appropriés par les élus car ils doivent également être envisagés comme des instruments de pilotage politique, pour donner à voir et maintenir le souffle et la volonté des décideurs. De ce point de vue, les indicateurs d'impact, par exemple, parlent peu aux décideurs. Le troisième point mis en avant par S. Depraetere est l'entrée en dialogue avec les parties 
concernées, comme pour d'autres collectivités inscrites dans des démarches d'évaluation, et les difficultés d'appropriation des enjeux du développement durable, qui sont des freins ; mais la région se doit de les surmonter, notamment dans le cadre de ses politiques contractuelles. Le quatrième point souligné par l'oratrice réside dans l'effet d'entraînement qui est aussi attendu: "surtout au niveau de nos politiques contractuelles, l'enjeu réside dans la communication, le fait d'être clair et simple, d'être compris ».

- Sandrine Rousseau a, pour sa part, tenté de mettre en perspective l'engouement actuel des acteurs publics pour l'évaluation en soumettant une réflexion à l'auditoire. Historiquement, les demandes d'évaluation émergent aux périodes de crise $(1929,1974 . .$. où il y a certain flou de l'action politique et de l'action publique. Ne s'agit-il pas d'un indice d'un certain désappointement des administrations face à des situations empreintes d'incertitudes qui les poussent à produire des méthodologies et des instruments de mesure ? La création de l'Indice du travail des femmes dans les années 1960 a ainsi été saluée comme une avancée importante dans le domaine de la statistique, mais n'a dans les faits rien changé aux pratiques... De ce point de vue, "évaluer n'est pas agir», et il apparait important de pouvoir étudier les "carrières sociales" des instruments d'évaluation (qui les produit, qui les utilise, que deviennent les résultats, quels sont leurs impacts éventuels sur la décision publique... ?) Il s'agit de construire des indicateurs sur lesquels il existe des moyens et une capacité d'action.

- André Colin, consultant et gérant de la coopérative Extra-Muros, a poursuivi cette réflexion en soulignant que l'enjeu principal n'est pas de construire un outil d'évaluation du développement durable, mais que les acteurs de l'organisation et/ou du territoire se l'approprient, l'utilisent, le fassent vivre et évoluer. Il a ainsi présenté rapidement les enjeux méthodologiques et opérationnels de la méthode d'accompagnement des collectivités proposée par Extra-Muros, actuellement utilisée pour l'accompagnement de l'élaboration de l'Agenda 21 des Communautés Urbaines de Nantes et de Brest. Cette méthode fondée sur la co-production par les acteurs du territoire, en croisant le plus possible les domaines de compétence, les points de vue et les métiers, ne conduit finalement pas à un consensus fade. " pour co-produire du neuf, il faut savoir prendre le risque de travailler avec des personnes avec qui nous n'avons pas forcement l'habitude de travailler ». On aboutit ainsi à des propositions et des exigences ambitieuses, qui ne sont pas sans amener parfois les élus à prendre des décisions marquées. Cette approche est issue du travail, "décisif pour nous ", mené par Hélène Combe avec la Communauté Urbaine de Dunkerque.

- La conclusion de cette table-ronde et de la matinée est revenue à Bruno Villalba, coanimateur du réseau DDTF, qui a présenté quelques éléments de réflexions sur l'appropriation des outils et les approches diverses du développement durable selon les acteurs et leurs besoins spécifiques, en prenant l'exemple de la Fédération des Centres Sociaux du Nord qui a engagé un travail de réflexion autour de la notion du développement durable pour ses propres activités. Comprendre la technicité des indicateurs, intégrer la dimension symbolique du développement durable - le très long terme - nécessitent une certaine capacité culturelle pour une traduction concrète. Les Centres Sociaux, au contact de populations socialement exclues, rencontrent des difficultés pour mettre en place le développement durable avec les bénévoles et les usagers de leurs Centres. On peut observer que ces populations ont une vision élaborée du développement durable (vision d'une inégalité spatiale très restreinte, prise en compte du 
court terme ...) qui fixe des priorités et une certaine hiérarchisation des «piliers": répondre aux besoins actuels, rechercher une équité intergénérationnelle basée le très court terme, qui peut se traduire en termes de mobilité individuelle notamment. Les méthodes démocratiques d'appropriation du développement durable sont ici au cœur de la problématique. Produire des indicateurs apparaît certes nécessaire, mais ils doivent, souligne B. Villalba, s'ancrer dans les pratiques quotidiennes et prendre en compte les visions et la parole des acteurs et en particulier des acteurs faibles (comme tous les « sans ", « sans papier ", « sans emploi » ...).

Bruno Villalba a, dans un second temps, insisté sur la systématisation des expérimentations dont un assez vaste aperçu a été proposé durant la matinée, et la professionnalisation, en termes de métiers, de compétences et d'instrumentation de cette question de l'évaluation au regard du développement durable. Dans les interventions, les indicateurs sont vus comme des démarches qualité (comment mieux faire?), or, il importe de ne pas évacuer la finalité de ces indicateurs: répondent-ils aux enjeux identifiés et aux problématiques soulevées dès Stockholm en 1972 ? Il existe une tension entre la standardisation du développement durable (qui se fait jour au travers des indicateurs en cours de construction) et les grandes finalités et les enjeux fondamentaux d'un modèle de développement durable (dont on fait appel en particulier à Rio en 1992).

\section{Etablir un diagnostic de territoire au regard du développement durable}

L'après-midi, animée par la consultante Corinne Blanchet, a été consacrée à différentes expériences méthodologiques, dont une opération menée par le CERDD en partenariat avec des formations de Master de différentes universités régionales sur l'élaboration de diagnostics territoriaux de développement durable. Marc Galochet, Maître de Conférences en géographie de l'Université d'Artois, a présenté les principaux résultats de ce travail. Différents groupes d'étudiants ont travaillé sur l'élaboration d'une méthodologie du diagnostic territorial, avec comme objectif final de réaliser un guide méthodologique, autour notamment de quatre territoires de projets. Si l'on considère que le diagnostic territorial est constitué de 6 étapes (phase préliminaire, phase de diagnostic, phase de conception, phase de mise en œuvre, phase d'évaluation ex post, phase de définition du programme d'action), comment concilier cette méthode avec la question du développement durable? Trois dimensions sont privilégiées : 1 ) un diagnostic partagé (qui vise à associer un maximum de partenaires à l'élaboration - mécanisme de co-production, co-élaboration - construction collective et progressive d'un programme dans un jeu continu de questions-réponses, en tenant compte des enjeux locaux), 2) un diagnostic global (le territoire est perçu dans son ensemble, en tenant compte des interactions, d'un élargissement du champ spatial - notamment dans une logique de coopération) et 3) un diagnostic stratégique (à partir de la connaissance approfondie du territoire, de ses opportunités et limites, on peut élaborer plusieurs scénarios de développement, afin de contribuer à une décision éclairée.

Le diagnostic prend la forme d'un document écrit, autour de trois éléments : un état des lieux partagé, une analyse croisée de l'existant et des potentialités de développement (qui permet de déterminer et de dégager les forces et les faiblesses, les opportunités et les freins potentiels en prenant en compte la totalité des effets d'une décision sur un 
territoire) et enfin, l'identification et la hiérarchisation des enjeux du territoire au regard du développement durable. Il convient de mettre en avant les enjeux propres du territoire en fonction des enjeux nationaux et internationaux du territoire.

La démarche de diagnostic se structure autour de trois phases: 1) préliminaire (élaboration d'un cahier des charges - précis mais évolutif - autour de l'équipe de projet) ; 2) pré-opérationnelle (mobilisation des moyens, humains, financiers et temporels - volet expertise et communication dans l'animation de la mission) ${ }^{15}$ et une phase de coproduction (avec les différents acteurs du territoire, ce qui permettra d'impulser/échanger l'information et partager les connaissances du territoire).

Ce diagnostic territorial doit permettre de fournir des scénarios stratégiques; il s'agit d'élaborer plusieurs scénarios stratégiques de développement durable mesurant les effets économiques, sociaux et environnementaux, ainsi que de réaliser une étude de faisabilité technique. C'est enfin un diagnostic qui permet de définir des pistes de suivi et d'évaluation (en définissant certains indicateurs, des programmes d'actions, etc. pour mesurer la pertinence territoriale et son efficacité) et installer une nouvelle pratique de la gouvernance territoriale. C'est donc une étape décisive dans l'élaboration d'un projet opérationnel.

Le document produit se veut avant tout pédagogique, afin d'inciter les acteurs locaux (porteur de projet, responsable de mission, etc.) à développer leurs projets locaux sur cette base préliminaire ${ }^{16}$.

\section{Comment appréhender l'évaluation du développement durable ? Éclairage sur des pratiques, des outils et des méthodes développés et expérimentés dans les collectivités}

51 Un Forum-débat a suivi cette première intervention de l'après-midi, animé par Corinne Blanchet ${ }^{17}$, avec Emmanuel Bertin (Animateur du Pays Cœur de Flandre) et Nathalie Holec (Chargée de mission au Conseil Régional NPDC, D2PE, mission animation et ingénierie du développement durable). Différents thèmes ont ainsi été abordés: à quoi sert une évaluation (pourquoi) ? Comment, en termes de méthode et d'outils?

\section{La grille de lecture développement durable du Pays Cœur de Flandre}

Emmanuel Bertin (animateur du Pays Cœur de Flandre) propose quelques réflexions autour de «La grille de lecture développement durable du Pays Cœur de Flandre: un référentiel local pour concevoir et analyser des actions au regard du développement durable ». Il s'agit d'un document qui vient juste d'être édité à la suite d'une année de travail. Cet outil s'inscrit pleinement dans la démarche de développement durable du Pays, plus particulièrement, de sa Charte de Pays qui constitue le projet de développement durable du territoire. La Charte vise à hiérarchiser les priorités dans le temps, face aux profonds bouleversements de ce territoire (démographie, conversion industrielle, etc.).

53 Un partenariat a été mis en place avec le CERDD en vue d'insuffler une dynamique d'information et de sensibilisation auprès des acteurs locaux en matière de 
développement durable. De là a résulté le besoin de construire un outil afin de faire progresser les projets locaux dans le sens du développement durable. Une grille a ainsi été bâtie par un groupe de travail réunissant des acteurs locaux avec l'appui technique du CERDD et du Pays. Une sorte de «boite à outils » a été produite, comprenant une grille de lecture et une fiche de synthèse des débats, une fiche mémento «structures ressources d'informations sur le développement durable». Soulignons que la DIREN Nord-Pas de Calais a apporté une contribution financière à la réalisation de la démarche.

Ce document, intitulé « Mon projet et le développement durable - Les bonnes questions à se poser en Pays Cœur de Flandre ", doit aider les acteurs locaux à concevoir des projets dans le sens du développement durable. Spécifique au territoire, l'outil vise à mixer les préoccupations globales liées au développement durable (lutte contre le changement climatique, maintien de la biodiversité ...) et les préoccupations locales de développement du territoire plus particulièrement identifiées dans la Charte de Pays.

La grille doit permettre l'émergence de questions principales, générales et spécifiques au territoire Cœur de Flandre. Elle vise ainsi à soutenir les différents porteurs de projets potentiels du territoire à intégrer les questionnements liés au développement durable et à articuler leurs actions avec les priorités stratégiques de développement durable du territoire.

Demain, tout projet sélectionné au titre du Contrat de Pays - et qui recevra donc des financements via le Contrat de Plan Etat-Région - passera automatiquement au tamis de la grille. La grille ne sera pas en tant que tel un outil de sélection des projets, car d'autres critères rentrent en lice. Le premier résultat attendu est de créer un réflexe, celui de s'interroger sur les valeurs et le sens qui fondent l'action. Le plus important est que les acteurs du territoire fassent un pas en avant vers le développement durable et que le développement durable soit accessible et compréhensible par le plus grand nombre. L'objectif est de faire connaître ces projets en faveur du développement durable et qu'ils se multiplient.

57 Un autre attendu, via la "fiche de synthèse des débats », est de mettre en évidence les principaux freins que rencontrent les acteurs du territoire pour mettre en œuvre le projet de développement durable. L'analyse de ces retours de terrain permettra ainsi au Pays d'interpeller les acteurs pertinents au bon niveau d'action pour lever les freins.

Il s'agit là d'un outil d'accompagnement des acteurs visant à rendre le développement durable compréhensible et pragmatique, à provoquer le débat et la discussion au sein d'un groupe de travail, d'un Conseil d'Administration, d'un Conseil Municipal, etc. Cependant, «l'urgence du quotidien" peut constituer un frein à l'utilisation de l'outil. L'outil met en avant, d'une part, la recherche de la double cohérence du projet avec la Charte et les critères du développement durable, et, d'autre part, l'amélioration continue du projet vers le développement durable.

\section{Le projet « indicateurs 21 » du Conseil régional Nord - Pas-de-Calais}

Nathalie Holec a présenté le projet « indicateurs 21 », inscrit dans l'Agenda 21 régional adopté en 2003. Ce projet fait partie des différentes réflexions et des différents chantiers engagés autour de l'évaluation du développement durable par le Conseil régional, et l'évaluation de l'agenda 21 régional prévue en 2006). Après un rapide rappel de l'origine 
de ce projet (la Stratégie de 2000 «Vers un développement durable en Nord - Pas-deCalais », les travaux du SRADT, et l'Agenda 21 régional), N. Holec présente la démarche :

- calculer l'empreinte écologique actuelle de la région; ce travail a été mené entre décembre 2002 et juin 2003 par un bureau d'études, et les résultats ont été présentés lors des Assises nationales du développement durable de Lille en juin 2003.

- calculer des indicateurs de développement humain ${ }^{18}$ à l'échelle de la région et en lien avec la région wallonne, chantier qui est en train de se conclure après deux années de travaux. Il a engagé une équipe de l'Université de Lille 1 conduite par le Pr. Jean Gadrey ${ }^{19}$, une équipe de l'Institut Wallon de l'évaluation, de la prospective et de la statistique (IWEPS), et la direction régionale de l'INSEE - avec le soutien du PNUD.

Les objectifs du Conseil régional étaient de pouvoir disposer d'indicateurs synthétiques alternatifs au PIB, de mesurer les progrès du territoire régional vers un développement durable, d'établir des comparaisons entre collectivités locales (précisément avec la Région Wallonne, partenaire du projet), de se doter d'outils de sensibilisation et de débat publics, et de disposer d'outils de pilotage des politiques publiques. Sur ce dernier objectif, il y avait une attente forte autour du calcul de l'empreinte écologique régionale, mais les acteurs régionaux ont été quelque peu déçus... S'il s'agit d'un outil pertinent de sensibilisation, cet indicateur n'est pas assez sensible aux changements des politiques publiques.

61 Au niveau des résultats, l'empreinte écologique de la région (5,40 hectares globaux par an et par habitant) est légèrement supérieure à celle de la France $(5,30 \mathrm{ha} / \mathrm{an} / \mathrm{hab}$.). Il faudrait ainsi sept territoires équivalents à la région Nord-Pas de Calais pour répondre aux besoins actuels (notamment en terme de transport et de consommation alimentaire) ${ }^{20}$ . Le calcul de l'IDH a été réalisé sur la période 1982-2003 pour les régions NPDC et Wallone, pour la France et la Belgique. L'IDH régional de 2003 est inférieur à celui national, et correspond au niveau national de 1993. Les indicateurs de Pauvreté Humaine (IPH) et de participation des femmes à la vie politique et économique ont été difficiles à élaborer, en raison du manque ou de la difficulté d'obtenir certaines données (variables sociales locales, absence de régionalisation des données statistiques, etc.), et ont nécessité des approximations pour permettre leur calcul.

Les résultats de ce chantier «Indicateurs 21 » doivent donner lieu à une publication au début de l'année 2006. Les autres perspectives sont la tenue d'ateliers décentralisés pour diffuser ces résultats et engager le débat avec les différents acteurs du territoire, et d'en faire des outils d'éducation populaire; encourager d'autres initiatives allant dans le même sens (dont le BIP 40, indicateur synthétique des inégalités socio-économiques), et travailler aux partenariats pour développer un tableau de bord du développement régional avec des indicateurs plus fins.

\section{Synthèse générale et suites à donner à la journée}

Après avoir remercié les organisateurs, et souligné la coopération entre ces acteurs, JeanFrançois Caron, Maire de Loos-en-Gohelle, Conseiller régional et ancien Vice-Président en charge du développement durable, de l'aménagement du territoire et de l'environnement ${ }^{21}$ insiste sur la confusion qui risque d'arriver dans la construction des indicateurs si l'on perd de vue la dimension spécifique du développement durable. Selon 
lui, les dimensions spécifiques du développement durable sont résumées par quatre principes ou aspects fondamentaux à prendre en compte :

- Partir des besoins (les indicateurs de démocratie participative sont au cœur de la problématique);

- L'approche transversale (comment les chercheurs comme les acteurs sortent de leurs approches disciplinaires ou sectorielles, et construisent une vision plus intégrée des problèmes et des solutions à proposer) ;

- L'emboîtement des échelles (local-global et réciproquement) ;

- La question du long terme et du moyen terme (notamment autour de l'enjeu de l'irréversibilité).

Sur la question des évaluations et des indicateurs, on ne peut évacuer la dimension technocratique; mais il faut tenir compte du fait que le mieux est l'ennemi du bien, et donc essayer de trouver un juste milieu, afin de construire des outils adaptables aux situations (il faut tenir compte d'une certaine souplesse et d'une capacité à s'adapter), de le faire dans une co-construction (ce qui complique encore le jeu), et, finalement, qu'importe l'indicateur s'il amène l'ensemble des acteurs à s'interroger sur les conséquences de leurs actes d'une part, et d'autre part, à s'inscrire dans une démarche qualité et à s'impliquer (une expertise ne sert à rien si elle n'est pas construite avec les personnes concernées - élus, populations, etc.) Il importe que chacun (élus, techniciens, citoyens...) ait un cheminement (on se pose des questions sur ses choix, ses actions au quotidien et avoir ainsi un réflexe d'évaluateur ...). Il s'agit d'insister sur ce qui est non durable, et de définir des priorités d'actions visant à éviter de telles situations. Une telle inversion de la démarche amène à un passage à l'action plus facilement opératoire.

Enfin, Jean-François Caron, qui est également Président du CERDD, précise que cette journée inaugure une nouvelle manière de travailler pour le CERDD (acteur expert), qui souhaite débattre de ce qui doit être fait avec le plus grand nombre : le développement durable est toujours une question de débats et d'arbitrages sur un territoire. Le rôle du CERDD est de créer les espaces de discussions et d'échanges, comme aujourd'hui avec le Réseau DDTF. Le CERDD va mettre en place en décembre 2005 une plate-forme collaborative (un «wiki») qui permettra de co-produire de la connaissance, d'instaurer un débat permanent, et progressivement de dégager une vision plus partagée, c'est une des façons de produire l'intelligence collective. La tenue d'autres journées d'informations et de travail de ce type sont à envisager prochainement. Pour conclure, J.-F. Caron formule «un rêve: celui de mettre en place le Groupe Régional des Acteurs du Développement Durable (GRADD) pour élargir les débats à l'ensemble de ceux qui se confrontent à la mise en œuvre du développement durable dans notre région et faire ses concrétisations ».

\section{En guise de conclusion subjective...}

Par B. Villalba et A. Goxe

Si le caractère évasif, peu stabilisé, de la notion de développement durable a été souligné (notamment autour des options «fortes » ou «faibles", mais aussi sur sa dimension «consensuelle» ou " pacificatrice»), on ne peut nier l'ampleur des effets pratiques qui peuvent lui être associés (voire imputés) depuis une dizaine d'année, tant dans les organisations privées que publiques: innovations institutionnelles, création d'outils 
d'évaluation, de méthodologie de projet, benchmarking ${ }^{22}$, etc. On note cependant une double hésitation : la première concerne la volonté de définir cette notion (peut-on, et est-ce souhaitable, de qualifier, définir, délimiter... le développement durable ?) et la seconde s'interroge sur la construction d'un référentiel commun (ou, d'une manière parfois plus pragmatique, l'élaboration d'une culture commune). À travers les exemples mobilisés autour de cette journée, on assiste à une procédure de formalisation progressive de cette notion à travers ses applications concrètes; de nombreuses illustrations sont liées aux questions environnementales, alors qu'il faut souligner la faiblesse des animations autour des questions économiques et sociales. De nombreuses réflexions ont aussi témoigné de l'importance à accorder à l'emboîtement des territoires et par conséquent à l'interaction de ces échelles territoriales (ce qui renforce la difficulté d'élaborer des méthodologies qui prendraient en compte la compréhension et la gestion de ces articulations entre les territoires).

Le déroulement des exposés ou table-ronde, les échanges - sans doute trop peu nombreux mais souvent constructifs avec la salle - montrent que nous assistons à l'émergence d'une culture professionnelle autour de cette thématique. À défaut d'élaborer une définition commune, cette journée témoigne que, dans certains champs professionnels - animateurs territoriaux, élus, consultants, universitaires, étudiants - apparaît un savoir relativement partagé, au point qu'il permet d'élaborer une réflexion sur les outils d'application du développement durable, sans que le temps soit consacré à une réflexion préalable à définir cette notion. Les exemples présentés, les profils professionnels présents, ont aussi offert un panel intéressant des acteurs de la professionnalisation de cette thématique. Que ce soit au niveau de certains cabinets spécialisés, de structures d'accompagnement, de postes salariés, ou bien encore de formations universitaires, nous avons eu l'occasion d'assister à une présentation assez représentative de ce qu'offre le développement durable des territoires comme orientations professionnelles (à la fois dans les registres rhétoriques, mais aussi les aptitudes professionnelles).

Il conviendrait cependant de prolonger cette réflexion, en travaillant aussi sur la manière dont se construisent les interprétations des résultats de ces évaluations. Une interprétation véhicule tout un ensemble de présupposés (grille de compréhension et d'analyse des résultats, etc.), qui, en trouvant une formulation plus distanciée lors de la rédaction d'un rapport final, trouvent une validation dans ce dispositif de restitution des évaluations.

70 Certaines réflexions ont mis l'accent sur les dimensions spécifiques de ces procédures d'évaluation au regard du développement durable. Pour le dire autrement, en quoi, certains des outils présentés au cours de la journée, favorisent-ils réellement la mise en place de politiques publiques différentes quant à leurs finalités ? À travers ces réflexions, nous voudrions insister sur la difficulté de ne pas dissocier une réflexion sur les instruments au détriment d'une réflexion politique sur le sens de ces outils ${ }^{23}$. Il a été ainsi rappelé, que dans une réflexion antérieure, Jacques Theys avait distingué un développement durable qui hésiterait entre le survivre ensemble et le mieux vivre ensemble. Que nous disent les instruments construits sur les choix implicites ou plus ou moins explicites qui ont présidé à leur élaboration?

71 Sans doute, cette première journée a montré, qu'alors même que la région Nord - Pas-deCalais et certains de ses partenaires locaux, sont considérés comme " pilotes » en France dans ce domaine, la culture de l'évaluation reste encore trop peu partagée, les analyses encore insuffisantes, particulièrement en ce qui concerne l'évaluation au regard du 
développement durable et la capitalisation des savoirs et des pratiques... Mais cette première journée a aussi démontré que des acteurs s'investissent, produisent et innovent en la matière. L'effort doit se poursuivre.

\section{NOTES}

1. Le Centre Ressource du Développement Durable (CERDD) est une émanation de l'Etat et de la Région Nord-Pas de Calais dont l'objectif est de faciliter l'application concrète du développement durable notamment dans les politiques publiques. L'activité du CERDD est structurée autour de trois missions principales: analyser, expertiser le développement durable; expliquer le développement durable et diffuser les bonnes pratiques; réunir les acteurs et animer des groupes d'échanges.

2. Le CERDD anime en effet un dispositif de sensibilisation renforcée et d'accompagnement auprès de 4 territoires de projets : le Pays du Calaisis, le Pays Cœur de Flandre, la Communauté d'Agglomération de la Porte du Hainaut et la Communauté Urbaine d'Arras. Au-delà des territoires directement concernés par le dispositif, les bénéficiaires de l'opération sont l'ensemble des territoires de projet du Nord-Pas de Calais. Parallèlement, le CERDD participe au Programme régional d'animation et de soutien aux Agendas 21 locaux (PRASA 21) lancé par le Conseil Régional Nord-Pas de Calais, notamment à travers le développement d'un réseau d'échanges entre acteurs impliqués dans la conception et la réalisation de démarches d'Agendas 21 locaux.

3. Le Centre de Développement des Eco-Entreprises (CD2E) a pour mission de favoriser la création et le développement d'activités dans le secteur de l'environnement. Il bénéficie du soutien de différentes institutions, dont le Conseil régional NPDC, l'ADEME, la DRIRE, la DRCE et la Commission Européenne.

4. Signalons ici son implication dans le cadre de la collaboration entre le CERDD et les universités régionales autour de diagnostic territorial à l'aune du développement durable, et ce, dans la perspective de l'élaboration de politiques territoriales de développement durable (agenda 21 local, agenda 21 de territoire). En effet, durant l'année universitaire 2004-2005, un travail d'étude et de réflexion a été mené avec 4 formations universitaires de la région Nord-Pas de Calais. A l'issue de ces travaux, un livret méthodologique sur le diagnostic territorial de développement durable a été co-produit avec pour objectif de capitaliser et de donner un sens global à ces travaux.

5. Ce service travaille sous la responsabilité du Délégué Interministérielle au Développement Durable, M. Christian Brodagh.

6. Constitué des hauts-fonctionnaires développement durable des différents ministères - HFDD de l'ADEME, des DIREN, de la MIES...)

7. A l'instar de feue la Commission Française du Développement Durable. Cf. l'avis n²002-07 sur « la culture et le développement durable ».

8. Cabinet Terr(e)itoires - Penser global, agir local; auteur d'une thèse soutenue en 2004 à l'ENMSE intitulée ; Le développement durable: penser le changement ou changer le pansement? Bilan et analyse des outils d'évaluation des politiques publiques locales en matière de développement durable en France. 
9. L'auteur a identifié au total une trentaine de démarche en cours (premier trimestre 2004) dont 26 OQADD effectivement réalisés.

10. Pour la plupart chargés de mission responsables du développement durable dans des organismes publics ayant mis en œuvre un OQADD ; enquête menée durant le premier semestre 2004. Une synthèse de l'analyse des OQUADD est disponible sur le site Internet du CERDD.

11. Godard (Olivier), «Le développement durable et le devenir des villes », Futuribles, ${ }^{\circ} 209$, mai 1996, pp 29-35.

12. Directeur du Centre de Prospective et de Veille Scientifique du Ministère de l'Equipement, des Transports et du Logement, ancien directeur scientifique de l'IFEN.

13. Agence régionale pour l'environnement de Midi-Pyrénées.

14. Direction de l'Animation de la Recherche, des Etudes et des Statistiques du Ministère de l'Emploi, de la Cohésion Sociale et du Logement.

15. Compilation des données et des connaissances sur la situation du territoire; identification des acteurs à associer à la démarche ; définition des critères de référence du DD, etc.

16. Le document est accessible sur le site extranet du CERDD : http://www.monDD.org et sur le wiki du CERDD http://wiki.cerdd.org

17. Consultante ABCD Durable

18. l'IDH (Indicateur de Développement Humain) du PNUD (combinaison du PIB/hab, de l'espérance de vie, et des taux de scolarisation (pour 1/3) et d'alphabétisation (pour 2/3) ; l'Indice de Pauvreté Humaine (IPH), l'Indice de participation des femmes à la vie politique et économique (IPF).

19. Jean Gadrey est Professeur Emérite de sciences économiques à l'Université de Lille 1. il est notamment l'auteur avec Florence Jany-Catrice d'un ouvrage de synthèse sur Les nouveaux indicateurs de richesse, Paris, La Découverte, coll. "Repères », 2005, et d'un rapport sur le même thème: Les indicateurs de richesse et de développement. Un bilan international en vue d'une initiative française. Rapport de recherche pour la DARES (2003).

20. Voir RÉGION NORD PAS-DE-CALAIS, 2004, Atlas régional du développement durable Nord-Pas-de-Calais, La Tour d'Aigues, éditions de l'Aube.

21. De 1998 à 2004.

22. eEurope, 2001, «eGovernment indicators for benchmarking eEurope », [online], http:// europa.eu.int/information_so ciety/eeurope/action_plan/pdf/ego vindicators.pdf; Le site de " benchmarking et réforme de l'Etat ", consultable à l'adresse : http://www.demlib.com/bench/ reformeetat

23. Lascoumes Pierre, Le Galès Patrick, (dir.), 2004, Gouverner par les instruments, Paris, Presses de Sciences Po, coll. « Gouvernance ».

\section{RÉSUMÉS}

Le 3 novembre 2005, le Centre Ressource du Développement Durable du Nord-Pas de Calais ( CERDD) et le réseau de recherche « Développement durable et territoires fragiles » organisaient une journée d'étude et de débat sur le thème: "Évaluer le développement durable: enjeux, méthodes, démarches d'acteurs ». Cette rencontre, qui bénéficiait du soutien du Conseil régional Nord-Pas-de-Calais, entendait contribuer à définir des outils d'aide à l'élaboration de politiques publiques locales de développement durable. Le présent article présente les principales 
réflexions menées, et tente un bilan de ces débats, qui ont permis une rencontre fructueuse entre les milieux universitaires et professionnels.

INDEX

Mots-clés : évaluation, prospective, cadre de référence, Nord-Pas de Calais

\section{AUTEURS}

BRUNO VILLALBA

Bruno Villalba est maître de conférences en sciences politiques, CERAPS, Université de Lille 2, bvillalba@univ-lille2.fr

\section{ANTOINE GOXE}

Antoine Goxe est ATER en sciences politiques, CERAPS, Université de Lille 2, antoinegoxe@revueddt.org

\section{JEAN-CHRISTOPHE LIPOVAC}

Jean-Christophe Lipovac est chargé de mission au Centre Ressource du Développement Durable du Nord-Pas-de-Calais, jclipovac@cerdd.org 